\title{
The Arapetic of the use of the Gum of Mascar in your Different Presentations to Minimize the Dream Inerance and Deprivation
}

\author{
Miranda Nava Gabriel ${ }^{1 *}$, María Elena Ramírez Bernal ${ }^{2}$ and Maria Odette Martí Torres ${ }^{3}$ \\ ${ }^{1}$ Head of the Neurology, Service of the Regional Military Specialty Hospital of Guadalajara, Mexico
}

${ }^{2}$ Internal Physician of the Regional Military Specialty Hospital of Guadalajara, Mexico

${ }^{3}$ Dentist and Specialist in Orthodontics and Maxillary Orthopedics, Autonomous University of the State of Mexico

*Corresponding author: Miranda Nava Gabriel, Head of the Neurology, Service of the Regional Military Specialty Hospital of Guadalajara, Mexico

\begin{abstract}
Introduction: Sleep is defined as the natural, periodic and reversible decrease in perception of the external environment, with the preservation of a certain degree of reactivity to the environment and autonomous functions. Sleep consists of 2 phases, REM phase and NREM phase, these phases alternate at night in the form of five to six cycles; of which the NREM phase is $75 \%$ presented and the REM by $25 \%$.

Thertita mark: Caffeine is a readily available short-acting stimulant that has been shown to reduce some of the deficits associated with sleep loss. With the rubber mark the absorption is carried out on the oral mucosa, which generates a greater bioavailability of the active substance and an immediate mechanism of action. The effective response dose can range from $100 \mathrm{mg}$ to $200 \mathrm{mg}$ and the effect arises from $6 \mathrm{~min}$ of administered the active substance. It is important to note that the dose of caffeine in chewing gum is directly proportional to the effects on sleep inertia, as well as the duration and maintenance of them.

Discussion: Being able to analyze the mechanisms of action of caffeine on sleep inertia, helps us to make a comparison of chewing gum with caffeine vs placebo (simple gum). Chewing generates for a short period of time the maintenance of performance on simple and complex tasks and improves alertness, with caffeine these same effects look prolonged for longer.

Conclusion: Caffeinated gum is an effective therapeutic presentation on sleep inertia. The dosage for a positive response ranges from 75 to $200 \mathrm{mg}$ depending on the circumstances of sleep restriction. That is, thanks to the effects generated by chewing on the state of alertness and cognition and the potentialization and duration of them by caffeine.
\end{abstract}

\section{Introduction}

Sleep disorders cause alterations in the quality of life of each and every patient. At least two-thirds of those with chronic degenerative diseases are affected by sleep disorders, the most common being the female sex.

Many individuals are subjected to night jobs where a constant state of alertness is required and scientifically proven how this affects the quality of life of each of the employees; for sleep is one of the most indispensable pillars of daily life, as it maintains critical aspects of cognition for optimal mental performance, as well as in mood, alertness and performance during working days and activities of the and everyday life. Awakening abruptly involves something known as "sleep inertia," which is characterized by impaired performance and subjective lack of alertness for a transitional period, which hardly progresses to a good cognition. It is well known that chewing facilitates concentration, maintains alertness and improves performance in cognitive tasks, but the question is how effective is by itself the effect of chewing a simple chewing gum vs to one that contains Caffeine? Therefore, one of the objectives of this research work, is to analyze the effects of chewing from a simple chewing gum to one that contains caffeine, verify the effectiveness by reversing the sleep inertia of each of 
them and know the mechanism of action, the pharmacokinetics, bioavailability and absorption of a caffeinated gum during sleep deprivation.

\section{Theoretical mark}

Normal sleep progresses in various stages: NREM (nonrapid eye movement) phase and REM (rapid eye movement) phase. These cycles alternate at night in the form of 5 to 6 cycles. In $75 \%$ of normal nighttime sleep is NREM and 25\% REM.

\section{Phase brake}

It happens every 90 minutes or so. It is characterized by zero muscle tone, presence of active sleep, as the electrical activity of the brain is maximum while the body is at full rest, rapid eye movements, heart rate as well as respiratory evidence of increased basal metabolism and the amount of gastric juice.

\section{Step nrem}

Also known as "deep sleep," it facilitates body rest and consists of 3 phases.

a) Phase N1 (Surface Sleep): very light sleep stage, lasting several minutes. It is characterized by a slight decrease in heart rate, breathing, muscle tone, general state of deep rest, relaxed and drowsy, maintaining active the ability to perceive external stimuli.

b) Phase N2 (Surface Sleep): characterized in EEG by sleep spindles and $\mathrm{k}$ complexes. Its duration is 10 to 15 minutes. At this stage the muscle tone relaxes further, slightly decreases body temperature and respiratory and cardiac rate, disappearing eye movements.

c) Phase N3 (Deep Sleep): Characterized by a global slowdown of the electrical path and the appearance of slow waves and high voltage (deltas) whose total duration must be greater than $20 \%$ and less than $50 \%$ of the plot. Sensory perception decreases markedly, as does heart and respiratory rates. Relaxation of the muscles is intensified. It is more difficult to wake up the subject, and if he does, he finds himself disoriented and confused. It is the fundamental stage for the subject to rest subjectively and objectively.

This research work was done based on various medical studies, where it is addressed from the rate of absorption, the bioavailability, the pharmacokinetics of caffeine in chewing gum, the effects of chewing, how is caffeine it reverses sleep inertia, assessing cognitive performance, mood and alertness after caffeinated gum is administered to the composition, formulation and design of said chewing gum. It will also include in a systematized way each and every one of the articles selected to carry out a bibliographic review and thus carry out a complete study of the effects and effectiveness of chewing gum with caffeine to minimize the inertia of sleep , based on scientific evidence from some experimental studies in which there is certainty of efficacy of it, since caffeine is involved in the autonomic nervous system and exerts its effects by acting as an adenosine receptor antagonist. The approach is an essential part of the daily life of the human being, since it is the pillar to be able to perform any cognitive activity, maintain an effective and consistent performance in order to successfully complete actions of daily life and working days. Fatigue can cause various complications and over time can affect the health of the individual, as well as leading to a reduction in efficiency during the day and the increase in the incidence of any type of accidents [1,2]. Caffeine is a white odorless powder that can have different molecular presentations, can range from an anhydride substance to contain a water molecule. Caffeine is a methylxanthine that inhibits the enzyme phosphodiesterase, generating an antagonistic effect on the central receptors of adenosine, this adenosine occurs during daily activities and binds to its receptors, generating a feeling of fatigue and consequently an induction into sleep. Due to the similarity of adenosine to caffeine, the latter takes place in adenosine receptors and thereby prevents the transmission of the fatigue signal generating that the person can continue performing his daily and work activities for a longer period, since contrary to the feeling of insomnia. Caffeine is a Central Nervous System (CNS) stimulant that can promote wakefulness and increase mental activity. In addition, it can stimulate the respiratory center, increase the frequency and depth of breathing and increase total muscle work. Caffeine is usually consumed and/or commonly administered in the form of a liquid substance, although there are other types of presentations such as tablets or capsules. A new way to consume caffeine is through chewing gum which in turn can provide additional advantages, some of them are:

a) Absorption is done through the oral mucosa, resulting in a greater bioavailability of the active substance at the systemic level.

b) Effective and immediate mechanism of action (oral mucosa).

c) It is easy to use and is suitable even for pediatric patients or patients with difficulty swallowing tablets or tablets.

d) It generates fewer side effects as the active substance is released in proportion to chewing.

e) The primary liver metabolism of the active substance is avoided, as they are absorbed directly by the oral mucosa.

f) Lower risk of overdose by chewing effect.

Chewing is a physiological motor activity involving many neural pathways, this action is associated with increased blood flow at the cerebral and orofacial level which in turn implies effectiveness in increasing alertness, physical well-being and improving memory performance. The active substance (caffeine) of chewing gum is released in proportion to chewing, this in turn is absorbed through the oral mucosa and another percentage of it is swallowed as a bolus with saliva, reaches the gastrointestinal tract and thanks to the caffeine is $s$ the absorption rate is faster compared to that of the tablet. In addition, it is important to mention that the control of the release of caffeine in chewing gum is for a long time and improves the variability of the release and retention times of the drug, being these other advantages of this new form administration of the Caffeine. 
It is important to note that the effectiveness of this chewing gum with the active substance which is caffeine, has to be argued based on studies that corroborate that effectiveness, such is the case of the P300 signal that is obtained thanks to an electroencephalogram, this signal is a neural record that is projected as a positive deflection and in turn measures the potential of the presence, magnitude, topography and duration of signaling of cognitive function. The signal is acquired more strongly around the parietal electrodes, although it has been suggested that there are also interactions between the frontal and temporal regions. Recent studies say that this P300 wave is composed of 2 secondary waves known as "P3A and P3B signals", these components respond individually to different stimuli and it has been suggested that the P3A wave originates in the frontal care mechanisms driven by stimuli during task processing, while $\mathrm{P} 3 \mathrm{~B}$ originates from parieto-temporal activity associated with attention and memory processing.

The potential related to the P300 signal had shortened latency after chewing gum, and the frontal and temporal beta power was increased by chewing the gum after performing a sustained attention task. The quantitative effects on the EEG of chewing gum without cognitive performance seem to be moderated by the taste, suggesting that chewing gum may alter alertness in the absence of cognitive performance. Working under pressure, i.e. in a setback, was associated with increased activity in the anterior cingulate cortex and left frontal convolution, where the motor neural regions of alertness and executive tasks are located [3]. This same effect was found when chewing gum without taste or smell, suggesting that the motor activity of chewing may be a key factor in explaining these results, however it is unclear whether a higher level of motor activity in chewing will increase associated effects, as there is evidence that a more vigorous chewing or a greater resistance to chewing does not moderate the effects on memory, the fact that chewing gum can increase arousal, it therefore reaches a peak and descends and it's while decreasing cognitive function in performing tasks that require attention (increasing heart rate and beta power during surveillance), this suggests that it is more plausible that more vigorous chewing can have a greater effect on attention to a short-term that in memory.

That is, chewing alone generates a potentization of the performance of a simple task as a result of a reflective motor activity that is generated in the body in the face of sleep deprivation, but this does not mean that this performance is maintained. One study shows that administering only $200 \mathrm{mg}$ of caffeine in conjunction with chewing gum, improves nothing more the performance of simple and complex activities and/or tasks, but also improves alertness compared to chewing without the active substance. In turn the period of pre-eyetion and cardiac autonomous activity remain unchanged during chewing with or without caffeine, generating a response reflected in the increase of parasympathetic activity with changes in rr intervals in the EEG, the latter mentioned above are predictors of the speed and accuracy in the most complex cognitive tasks during sleep deprivation, alertness and performance maintenance during the realization of them.
Some other studies show evidence that the use of caffeine in chewing gum is also equally effective in dispensing sleep in the face of a post siesta; in a double-blind study with 15 adults as participants they were given this chewing gum containing $100 \mathrm{mg}$ of caffeine at the hour and 6 hours after waking up vs. placebo, they were subsequently assigned psychomotor tasks before a watch dog at $0,6,12$ and 18 minutes. The rating of the tests was carried out based on the response rate and the number of hits during the tests. Among the results it was observed that $100 \mathrm{mg}$ of caffeine did not fully restore performance, but showed an improvement in response rate by $85 \%$ compared to placebo $73 \%$; since the effect of caffeine was evident at 6 min after waking up, which continued to improve performance until 18 minutes, i.e. the results indicate that $100 \mathrm{mg}$ of caffeine substantially attenuates sleep inertia in the face of a sudden awakening by a time-values period, but higher doses (200 $\mathrm{mg}$ ) are expected to more easily antagonize sleep inertia as a whole for a longer prolonging period.

\section{Discussion}

The purpose of this article is to evaluate based on a collection of articles the efficacy of caffeine as an active substance in a new therapeutic presentation vs placebo, studying the pharmacokinetics, bioavailability and half-life of caffeine. Among the bibliographic reviews that were addressed for the realization of this article, it is evidence that caffeine in chewing gum is a novel and new presentation that is effective in counteracting sleep inertia, improving alertness, cognition and maintaining performance during the simple and complex activities of daily life. It is important to note that the effects of caffeine are directly proportional to the dose administered in patients as well as on the route of administration. Chewing a gum without the active substance (placebo) generates a later maintenance of performance as well as alertness with the difference that these effects occur over a very short period of time.

\section{Conclusion}

Caffeine is an odorless powder that inhibits the enzyme phosphodiesterase, which generates an antagonistic effect on the central receptors of adenosine resulting in antagonism of the transmission of the fatigue signal, promoting wakefulness and increasing mental activity. The effect of chewing is associated with increased orofacial blood flow that increases alertness, physical well-being and memory performance. A combination of chewing gum with caffeine enhances and synergizes the effects that chewing alone generates, resulting in a new, novel and effective therapeutic presentation on sleep inertia, since it is easy to use and its absorption is done through the oral mucosa, which generates a greater bioavailability of the active substance and an immediate mechanism of action, in turn those effects are present for a prolonged period since the caffeine inside the gum is released in a way proportional to chewing. Caffeine can provide better alertness and performance at doses of 75 to $150 \mathrm{mg}$ after acute sleep restriction and at doses of 200 to $600 \mathrm{mg}$ after one night or more sleep loss. Caffeine is unlikely to have negative effects on sleep that follows 8 hours or more after administration. However, frequent use of caffeine can lead toilet and withdrawal syndrome. 


\section{References}

1. Emsley J (2004) Vanity, vitality and virility. The science behind the products you love to buy. Oxford University Press, New York, USA.
2. McKenzie D (1993) Chewing gum gave Stone Age punk a buzz. New Scientist.

3. Weaver V (2003) Wrigley patents anti impotence gum.
(C) (P) This work is licensed under Creative

To Submit Your Article Click Here: Submit Article

DOI: $10.32474 / 0 J N B D .2019 .03 .000164$

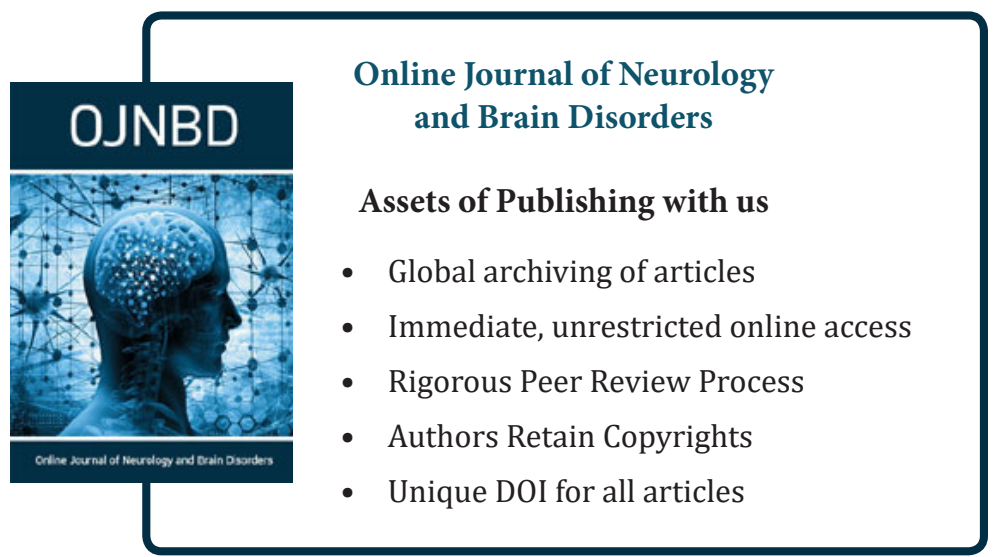

